\title{
Systematische Identifizierung energieeffizienter Antriebskonfigurationen in Elektrofahrzeugen
}

\author{
Shaohui Yuan (D) - Wilfried Hofmann
}

Eingegangen: 3. Dezember 2021 / Angenommen: 1. Februar 2022 / Online publiziert: 8. März 2022

(C) Der/die Autor(en) 2022

\begin{abstract}
Zusammenfassung In der Projektierungsphase eines Elektrofahrzeugs entstehen optimale Antriebskonfigurationen erst durch die gezielte Kombination der einzelnen Antriebskomponenten. Um technisch vorteilhafte Auslegungszusammenhänge zu erkennen, ist eine systematische Untersuchung der Auslegungsfreiheitsgrade erforderlich. Diese Freiheitsgrade ergeben sich v.a. aus der Wahl der Auslegungsparameter, der Komponententypen und der Antriebstopologien. In diesem Artikel wird gezeigt, wie bereits in einer frühen Entwicklungsphase energieeffiziente Antriebskonfigurationen für Elektrofahrzeuge durch analytische Methoden identifiziert werden. Diese Methoden basieren grundsätzlich auf physikalischen Modellen und frei zugänglichen Datenblattangaben der Komponenten, die v. a. Energiespeicher, Wechselrichter, Elektromotor und Getriebe umfassen. Mithilfe dieser Methoden lässt sich der energetische Vergleich der zahlreichen Antriebskonfigurationen ohne großen Zeitaufwand durchführen. Die Ergebnisse führen schließlich zu einem informativen Auswahldiagramm, mit welchem aussagekräftige Prognosen zu den energieeffizienten Konfigurationen für beliebige Fahrprofile getroffen werden.
\end{abstract}

Schlüsselwörter Energieeffizienz · Antriebsstrang · Elektrofahrzeug · Auslegungsmethodik · Fahrprofil

\section{S. Yuan $(\bowtie) \cdot$ W. Hofmann}

Elektrotechnisches Institut, Professur für Elektrische

Maschinen und Antriebe, Technische Universität Dresden,

Helmholtzstraße 9, 01069 Dresden, Deutschland

Shaohui.Yuan@tu-dresden.de

\section{Systematic identification of energy-efficient powertrain configurations in electric vehicles}

Abstract Optimal powertrain configurations develop only through the specific combination of individual powertrain components during the project planning phase of an electric vehicle. In order to identify the technically beneficial design correlations, a systematic investigation of the degrees of freedom in the powertrain design is necessary. The degrees of freedom arise primarily from the selection of design parameters, component types, and drive topologies. This article shows how energy-efficient powertrain configurations of electric vehicles can be identified at an early stage of development using analytical methods. These methods are based on physical models and freely available component data sheets. The components include a battery, inverter, electric motor, and gearbox. With these methods, the energy efficiency of various powertrain configurations can be evaluated quickly. The evaluation leads to an informative selection diagram, which allows forecasting energy-efficient configurations for different driving profiles.

Keywords Energy efficiency · Powertrain · Electric vehicle $\cdot$ Design methodology $\cdot$ Driving profile

\section{Einleitung}

Ein unbestrittener Vorteil von Elektrofahrzeugen gegenüber Verbrennerfahrzeugen ist hohe Energieeffizienz. Ein modernes Elektrofahrzeug hat typischerweise einen Batterie-to-Wheel-Wirkungsgrad von 70-80\%, während der Tank-to-Wheel-Wirkungsgrad eines Verbrennerfahrzeugs bei nur 20-25\% liegt. Dieser Vorteil von Elektrofahrzeugen wird jedoch durch die im Vergleich mit fossilen Kraftstoffen viel geringere Energiedichte von Akkumulatoren häufig übersehen. Trotz 
des erheblich höheren Wirkungsgrads des elektrischen Antriebsstrangs wird die Reichweite von Elektrofahrzeugen aufgrund der begrenzten mitgeführten Energiemenge stark eingeschränkt. Für Elektrofahrzeuge sind größere Batteriekapazität und effizientere Antriebsstränge in absehbarer Zeit noch zwei vielversprechende Wege zur Verminderung von nachteiligen Auswirkungen einer geringen Batterie-Energiedichte.

In den letzten Jahren werden immer mehr Elektrofahrzeuge mit größerem Batteriepack auf den Markt gebracht. Der Einsatz größeren Batteriepacks kann zwar die Reichweite eines Elektrofahrzeugs erhöhen, erhöht aber auch die Gesamtkosten und den spezifischen Energieverbrauch durch die damit verbundene Gewichtserhöhung. Im Gegensatz dazu kann die Effizienzerhöhung nicht nur eine verlängerte Reichweite ermöglichen, sondern sich auch auf die Einsparung von Betriebskosten, Gewicht und Bauvolumen vorteilhaft auswirken. Ein verlustarmer Antriebsstrang in Elektrofahrzeugen verursacht weniger Verlustwärme und kann damit zu einem kleiner dimensionierten Kühlsystem führen, welches wiederum zur Reduzierung des gesamten Energieverbrauchs beitragen kann.

Ein typischer elektrischer Antriebsstrang besteht aus mehreren Antriebskomponenten wie Batterie, Wechselrichter, Elektromotor und Getriebe. Die Wirkungsgrade der einzelnen Komponenten werden in der Regel durch eine gezielte Optimierung im Entwurf [1] bzw. durch Steuerungsstrategien [2] positiv beeinflusst. Aus Sicht des gesamten Antriebsstrangs bilden die einzelnen Komponenten mit dem höchsten Wirkungsgrad jedoch nicht immer das energieeffizienteste System, da hinsichtlich der Systemeffizienz die Synergie zwischen den einzelnen Komponenten vielmehr eine entscheidende Rolle spielt. Eghtessad zeigt in [3] das Potenzial zur Reduzierung des Energieverbrauchs durch die ganzheitliche Optimierung der Parameter wie z.B. Speicherkapazität, Motorleistung und Getriebeübersetzung. Neben Parameteroptimierung zeigt die Untersuchung von Reupold [4], wie sich die Wahl der Antriebstopologien und der Komponententypen auf die Energieeffizienz eines elektrischen Antriebsstrangs auswirken kann. Ähnlich wie die in [3] und [4] beschriebenen Methoden wird aktuell die energetische Optimierung des Antriebsstrangs meistens für ein bestimmtes Fahrprofil durchgeführt. Dies bringt allerdings ein Problem mit sich, dass die für ein Fahrprofil energieoptimale Antriebskonfiguration für andere Einsatzprofile durchaus ineffizienter ist. In diesem Beitrag wird ein Systemansatz vorgestellt, um fahrprofilübergreifende Aussagen für die Konfigurationsauswahl zu treffen. Kern der Methode ist eine analytische Verlustabschätzung des Antriebsstrangs und ein neuartiges Auswahldiagramm. Basierend auf physikalischen Modellen der Antriebskomponenten kann die Verlustwärme der einzelnen Komponenten unter Berücksichtigung von Steuerungsstrategien berechnet werden. Das Auswahldiagramm dient als ein informatives Mittel zur Darstellung der Verteilung der energieeffizienten Antriebskonfigurationen.

\section{Allgemeine Auslegungskriterien elektrischer Antriebsstränge}

Der Elektroauto-Markt wächst in den letzten Jahren weltweit rasant, insbesondere in China und Deutschland. Damit kommen immer mehr neue Fahrzeugmodelle für vielfältige Anwendungen auf den Markt. Eine klare Marktpositionierung stellt eine bedeutende Voraussetzung für ein erfolgreiches Fahrzeugmodell dar. Hinsichtlich der Marktpositionierung sind angemessene Randbedingungen für Fahrzeugeigenschaften zu definieren, die etablierten Firmenphilosophien genügen und gleichzeitig gesetzliche Vorschriften erfüllen.

\section{Gesetze, Normen und Standards}

Für konventionelle Fahrzeuge und Elektrofahrzeuge bestehen aktuell zahlreiche nationale und internationale Gesetze, Normen und Standards. Die meisten davon beschreiben die Anforderungen hinsichtlich Fahrzeugsicherheit und standardisieren die Messbzw. Testverfahren. Diese sind zwar wichtig für die Fahrzeugentwicklung, haben aber keine direkte Wirkung auf die Dimensionierung des Antriebsstrangs. Für die Dimensionierung des Antriebsstrangs interessant sind staatliche Richtlinien in den Bereichen Straßenverkehr und Straßenbau.

- China: Technical Standard of Highway Engineering

- Deutschland: Richtlinien für die Anlage von Stadtstraßen, Landstraßen, Autobahnen

Aus diesen Richtlinien sind Informationen hinsichtlich Geschwindigkeitsbegrenzung (Tab. 1) und Straßensteigung (Abb. 1) zu entnehmen. Diese Kenngrö-

\section{Tab. 1 Autobahn-Geschwindigkeitsbegrenzung PKW

\begin{tabular}{lll} 
& China & Deutschland \\
\hline Min & $60 \mathrm{~km} / \mathrm{h}$ & $60 \mathrm{~km} / \mathrm{h}$ \\
\hline Max & $120 \mathrm{~km} / \mathrm{h}$ & $\begin{array}{l}130 \mathrm{~km} / \mathrm{h} \\
\text { (Richtgeschwindigkeit) }\end{array}$ \\
\hline
\end{tabular}

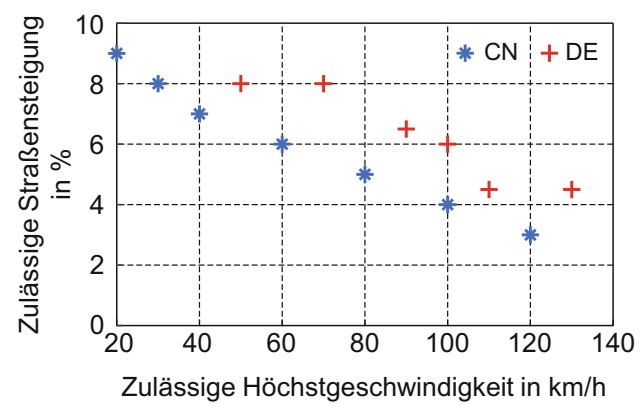

Abb. 1 Zulässige Straßensteigung hinsichtlich der Geschwindigkeit 


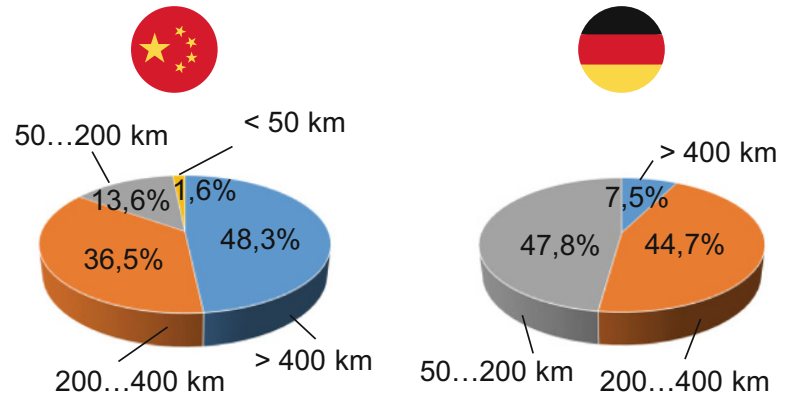

Abb. 2 Anteil der Befragten bzgl. der gewünschten Reichweite laut $[5,6]$

ßen stellen Mindestanforderungen für ein alltagstaugliches Fahrzeug dar.

Eine weitere relevante Norm ist die ISO/PAS 19295, welche die Spannungsklasse der Energiespeicher in Elektrofahrzeugen festgelegt. Dabei werden mehrere Unterklassen (220 V, 420 V, $750 \mathrm{~V}$ usw.) definiert, deren Werte sich grundsätzlich an der Sperrfähigkeit moderner Halbleiterbauelemente orientieren.

\section{Kundenerwartungen an die Reichweite}

Neben den Fahreigenschaften spielt die Reichweite eines Elektrofahrzeugs häufig eine noch wichtigere Rolle für die Kaufentscheidung der Kunden. Aus den Studien [5-8] geht hervor, dass die meisten Nutzer von Elektrofahrzeugen eine viel höhere Reichweite (Abb. 2) wünschen, als für die tägliche Nutzung nötig (Tab. 2).

Es ist einerseits darauf zurückzuführen, dass die tatsächliche Reichweite eines Elektrofahrzeugs je nach Fahrweise und Umgebungstemperatur viel weniger als die von den Herstellern angegebenen Werte sein kann. Andererseits wollen die Nutzer alle geplanten Fahrten bequem ausführen können, ohne sich sorgen zu müssen, liegenzubleiben.

\section{Fahrzeugklassebedingte Unterschiede in den Auslegungsanforderungen}

Um einen Antriebsstrang zu dimensionieren, werden einige Ausgangsparameter wie Fahrzeugabmessungen, Fahrzeugmasse und erforderliche Fahrleistungen benötigt. Für konventionelle Fahrzeuge werden diese Parameter aus den vergangenen Fahrzeugmodellen abgeschätzt. Aufgrund der Unterschiede zwischen konventionellen und elektrischen Antriebssträngen und der fehlenden langjährigen Erfahrung in der Entwicklung besteht bei Elektrofahrzeug die Schwierigkeit, diese Ausgangsparameter für ein neues Fahr-

Tab. 2 Reale Tagesnutzung der Fahrzeuge nach [7, 8] $95 \%$ der Pkw-Fahrten im Alltagsverkehr

\begin{tabular}{l|l}
\hline China & Deutschland \\
$<80 \mathrm{~km}$ & $<50 \mathrm{~km}$ \\
\hline
\end{tabular}

Tab. 3 Referenzwert der Fahrzeugparameter

Fahrzeug-Leergewicht in $\mathbf{k g}$

\begin{tabular}{l|l|l|l|l|l|l}
- & Gruppe 1 & \multicolumn{3}{c}{ Gruppe 2 } & \multicolumn{2}{l}{ Gruppe 3 } \\
\hline- & V-Fzg & E-Fzg & V-Fzg & E-Fzg & V-Fzg & E-Fzg \\
\hline Min & 900 & 1095 & 1206 & 1450 & 1395 & 1611 \\
\hline Med & 1099 & 1235 & 1425 & 1624 & 1715 & 1847 \\
\hline Max & 1325 & 1577 & 1705 & 1725 & 2335 & 2250 \\
\hline Fahrzeug-Motorleistung in kW & & & \\
- & Gruppe 1 & Gruppe 2 & Gruppe 3 \\
\hline - & V-Fzg & E-Fzg & V-Fzg & E-Fzg & V-Fzg & E-Fzg \\
\hline Min & 44 & 30 & 70 & 70 & 88 & 202 \\
\hline Med & 74 & 61 & 103 & 103 & 200 & 328 \\
\hline Max & 147 & 135 & 331 & 160 & 470 & 592
\end{tabular}

Fahrzeug-Beschleunigungszeit in s

\begin{tabular}{|c|c|c|c|c|c|c|}
\hline \multirow{2}{*}{ - } & \multicolumn{2}{|c|}{ Gruppe 1} & \multicolumn{2}{|c|}{ Gruppe 2} & \multicolumn{2}{|c|}{ Gruppe 3} \\
\hline & $V-F z g$ & $E-F z g$ & $V-F z g$ & E-Fzg & $V-F z g$ & $E-F z g$ \\
\hline Min & 6,5 & 6,9 & 4 & 7,5 & 2,6 & 2,6 \\
\hline Med & 12,1 & 11,9 & 8,3 & 10,1 & 5,7 & 4,5 \\
\hline Max & 16,3 & 15,9 & 13,5 & 13,7 & 12,6 & 5,9 \\
\hline \multicolumn{7}{|c|}{ Fahrzeug-Höchstgeschwindigkeit in km/h } \\
\hline- & \multicolumn{2}{|c|}{ Gruppe 1} & \multicolumn{2}{|c|}{ Gruppe 2} & \multicolumn{2}{|c|}{ Gruppe 3} \\
\hline- & $V-F z g$ & $E-F z g$ & $V-F z g$ & $E-F z g$ & $V-F z g$ & $E-F z g$ \\
\hline Min & 160 & 105 & 170 & 130 & 193 & 180 \\
\hline Med & 177 & 130 & 215 & 140 & 237 & 230 \\
\hline $\operatorname{Max}$ & 240 & 160 & 280 & 160 & 315 & 262 \\
\hline
\end{tabular}

zeugmodell abzuschätzen. Aus diesem Grund ist eine statistische Untersuchung zur Identifizierung der typischen Fahrzeugparameter für Elektrofahrzeuge sinnvoll. Tab. 3 zeigt die Referenzwerte basierend auf einer Statistik. In die Statistik wurden ca. 350 Fahrzeugvarianten von 99 Fahrzeugmodellen einbezogen.

Diese Fahrzeugmodelle umfassen unterschiedliche Fahrzeugklassen und werden in drei Gruppen klassifiziert:

- Gruppe 1: Kleinst- und Kleinwagen

- Gruppe 2: Kompaktklasse

- Gruppe 3: Mittel- bis Oberklasse

Die Abkürzung V-Fzg und E-Fzg steht jeweils für Fahrzeuge mit Verbrennungsmotor und Fahrzeuge mit Elektromotor. Auffällig ist der Unterschied zwischen V-Fzg und E-Fzg bzgl. der Höchstgeschwindigkeit. Für Elektrofahrzeuge der Gruppe 1 und 2 ist die Höchstgeschwindigkeit um ca. ein Drittel niedriger als für Verbrenner der gleichen Fahrzeugklasse. Der Hauptgrund ist das verwendete Getriebe im Antriebsstrang. Für Elektrofahrzeuge der Kleinst- bis Kompaktklasse ist aktuell das 1-Gang-Getriebe das gebräuchliche Getriebekonzept. Aufgrund der festen Übersetzung kann ein Kompromiss zwischen Anfahrsteigfähigkeit und Höchstgeschwindigkeit nicht umgangen werden. Im Vergleich zu der Gruppe 1 und 2 kann die Höchstgeschwindigkeit bei Elektrofahrzeugen der Oberklasse durch die Nutzung eines 2-Gang-Getriebes bzw. der Mehr-Motoren-Antriebe etwas verbessert werden. 


\section{Verlustabschätzung eines elektrischen Antriebsstrangs}

Für die Verlustabschätzung eines elektrischen Antriebsstrangs, der aus Batterie, Wechselrichter, Elektromotor und Getriebe besteht, kommen häufig stationäre Verlustmodelle der Komponenten zum Einsatz. Dabei werden zur Parametrisierung dieser Modelle physikalische Parameter der Komponenten benötigt. Aufgrund der wettbewerbsbedingten Geheimhaltung legen viele Komponentenhersteller allerdings die Konstruktionsdetails nicht offen. Deswegen wird im Folgenden ein Verfahren vorgestellt, mit dem die für Modellbildung relevanten Parameter aus nur wenigen grundsätzlichen Datenangaben abgeschätzt werden.

\section{Verlustleistung der Batterie}

Die Verlustabschätzung des Antriebsstrangs kann anhand einer Rückwärtssimulation durchgeführt werden. Aus Drehmomenten und Drehzahlen an den Fahrzeugrädern werden Leistungen und Verlustleistungen von Getriebe, Motor, Wechselrichter bis zu Batterie nacheinander ermittelt. In der Praxis ist eine gleichmäßige Nutzung aller Batteriezellen in einem Batteriepack immer anzustreben, damit alle Zellen ungefähr gleichzeitig das Lebensdauerende erreichen. Deswegen wird bei der Berechnung angenommen, dass die Leistung des Batteriepacks auf alle Zellen gleichmäßig verteilt wird.

Unabhängig von der konkreten Verschaltung der Batteriezellen kann die Verlustleistung einzelner Zellen mit einem vereinfachten Ersatzschaltbild in Abb. 3 berechnet werden. Dabei ist $U_{\mathrm{OCV}}$ die Zellen-Leerlaufspannung. $R_{\mathrm{i}}$ ist der Zellen-Innenwiderstand und verursacht die Verlustleistung der Batteriezelle. Die Größen der Leerlaufspannung und des Innenwiderstands sind normalerweise in Datenblättern angegeben und lassen sich auch anhand von [9] aus den Entladekennlinien der Batteriezelle abschätzen.

\section{Verlustleistungen des Wechselrichters}

In einem Wechselrichter werden die Verlustleistungen vor allem durch die Halbleiterbauelemente hervorgerufen. Dazu zählen die Durchlassverluste und die Schaltverluste. Im Vergleich mit diesen Verlusten ist der Leistungsbedarf der Gate-Treiber relativ gering

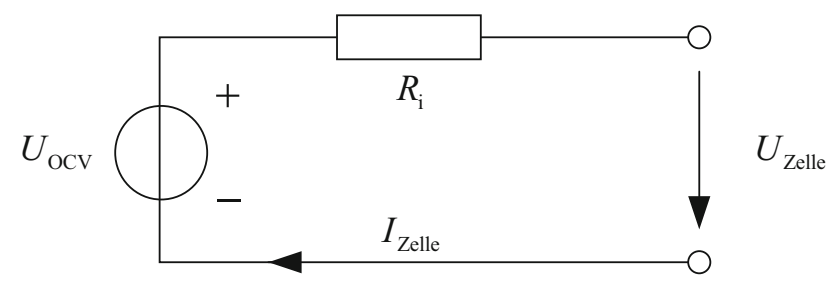

Abb. 3 Ersatzschaltbild der Batteriezelle und kann bei der Berechnung vernachlässigt werden [10].

Für einen 2-Level-Wechselrichter werden alle sechs Transistoren bzw. alle sechs Dioden identisch belastet. In einer Grundschwingungsperiode werden die mittlere Durchlassverlustleistung $P_{\mathrm{c}}$ eines Transistors bzw. einer Freilaufdiode anhand von [11] allgemein mit der Gl. 1 beschrieben. $u_{\text {on }}$ ist der Spannungsabfall an einem eingeschalteten Transistor bzw. Diode und abhängig von der Schleusenspannung $U_{0}$, dem differentiellen Widerstand $r_{\mathrm{on}}$ und dem Durchlassstrom $i_{\mathrm{c}}$.

$$
\begin{aligned}
& P_{\mathrm{c}}=\frac{1}{2 \pi} \cdot \int_{0}^{\pi} \frac{t_{\mathrm{on}}}{T_{\mathrm{P}}} \cdot u_{\mathrm{on}}(\omega t) \cdot i_{\mathrm{c}}(\omega t) \mathrm{d} \omega t \\
& i_{\mathrm{c}}=\hat{i}_{\mathrm{c}} \cdot \sin (\omega t) \\
& u_{\mathrm{on}}=U_{0}+r_{\mathrm{on}} \cdot i_{\mathrm{c}}
\end{aligned}
$$

Bzgl. einer Pulsperiode $T_{\mathrm{P}}$ ist die Pulsdauer eines Transistors $t_{\mathrm{on}, \mathrm{T}}$ bzw. einer Diode $t_{\mathrm{on}, \mathrm{D}}$ abhängig von dem Modulationsverfahren und den Betriebsparametern wie Modulationsgrad $m$ und Phasenwinkel $\varphi$. Für Sinus-Modulation bzw. Rauzeigermodulation, die gebräuchliche Modulationsverfahren in Traktionsumrichtern sind, ergeben sich die Pulsdauern aus Gl. 4 und (5).

$$
\begin{aligned}
& t_{\mathrm{on}, \mathrm{T}}=\frac{T_{\mathrm{P}}}{2} \cdot[1+m \cdot \sin (\omega t+\varphi)] \\
& t_{\mathrm{on}, \mathrm{D}}=\frac{T_{\mathrm{P}}}{2} \cdot[1-m \cdot \sin (\omega t+\varphi)]
\end{aligned}
$$

Die Schaltverluste sind proportional zur Schaltfrequenz $f_{\mathrm{s}}$ und können prinzipiell über $i_{\mathrm{c}}$ und der Zwischenkreisspannung $U_{\mathrm{ZK}}$ linear approximiert werden. In Gl. 6 und (7) sind $E_{\text {on }}$ und $E_{\text {off }}$ die Schaltwärme für das Ein- und Abschalten der Halbleiterbauelemente bei einer Referenzspannung $U_{\text {Ref }}$ und einem Referenzstrom $I_{\text {Ref. }}$

$$
\begin{aligned}
& P_{\mathrm{sw}, \mathrm{T}}=\frac{f_{\mathrm{s}} \cdot\left(E_{\mathrm{on}, \mathrm{T}}+E_{\mathrm{off}, \mathrm{T}}\right)}{\pi} \cdot \frac{\widehat{i_{\mathrm{c}}}}{I_{\mathrm{Ref}}} \cdot \frac{U_{\mathrm{ZK}}}{U_{\mathrm{Ref}}} \\
& P_{\mathrm{sw}, \mathrm{D}}=\frac{f_{\mathrm{s}} \cdot E_{\mathrm{off}, \mathrm{D}}}{\pi} \cdot \frac{\widehat{i_{\mathrm{c}}}}{I_{\mathrm{Ref}}} \cdot \frac{U_{\mathrm{ZK}}}{U_{\mathrm{Ref}}}
\end{aligned}
$$

Die Einschaltverluste der Diode können meistens vernachlässigt werden, da diese Verluste normalerweise nur wenige Prozent der Abschalt- und Durchlassverluste betragen. Die für Verlustberechnung notwendigen Halbleiter-Parameter sind in der Regel in den standardisierten Datenblättern von HalbleiterHerstellern angegeben. Die betriebsabhängigen Parameter wie Durchlassstrom und Modulationsgrad müssen seitens der elektrischen Maschine abhängig von Motorsteuerungsverfahren bestimmt werden.

\section{Verlustabschätzung der elektrischen Maschine}

Anders als für Industrieanwendungen werden Traktionsmotoren für Elektrofahrzeuge nicht direkt aus Pro- 
Stator-Entwurf

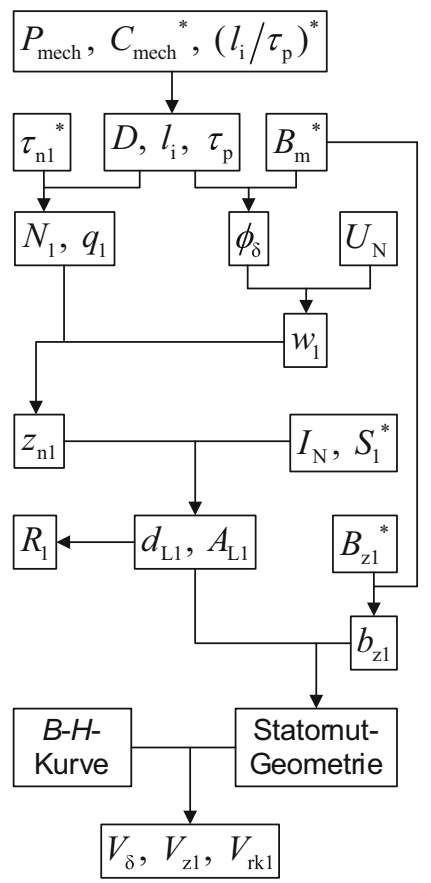

Abb. 4 Grobentwurf einer Asynchronmaschine

duktkatalogen von Motorherstellern ausgewählt, sondern je nach Fahrzeugkonzepten speziell ausgelegt. Deswegen ist in der Konzeptphase eine zuverlässige Abschätzung von Motorparametern und damit Motorverlusten für den Konzeptvergleich sehr wertvoll. Um eine Betrachtung eines möglichst breiten Spektrums von Leistungsklassen $\mathrm{zu}$ ermöglichen, werden relevante Motorparameter ausgehend von einem generellen Motorentwurf abgeschätzt, der grundsätzlich auf dem analytischen Entwurfsprozess nach Vogt [12] basiert. In dieser Arbeit wird das Abschätzungsverfahren für die Asynchronmaschine (ASM) weiter erklärt.

In Abb. 4 wird der Entwurfsprozess einer Asynchronmaschine mit Käfigläufer dargestellt. Für die Parameter, die mit * gekennzeichnet sind, sollten zuerst Richtwerte anhand von [12] ausgewählt und danach durch Nachrechnung bestätigt werden. Der

Tab. 4 Symbole im Motor-Grobentwurf

\begin{tabular}{|l|l|l|l|}
\hline$A_{\mathrm{L}}$ & Leiterquerschnitt & $P_{\text {mech }}$ & Leistung \\
\hline$A_{n}$ & Nutfläche & $q$ & Lochzahl \\
\hline$A_{\mathrm{r}}$ & Ringquerschnitt & $R$ & Widerstand \\
\hline$B$ & Induktion & $S$ & Stromdichte \\
\hline$b_{2}$ & Zahnbreite & $U$ & Spannung \\
\hline$C_{\text {mech }}$ & Ausnutzungsfaktor & $W$ & Windungszahl \\
\hline$D$ & Bohrungsdurchmesser & $V$ & Magnetischer Spannungsabfall \\
\hline$d$ L & Drahtdurchmesser & $Z_{n}$ & Leiterzahl je Nut \\
\hline I & Strom & $\tau_{p}$ & Polteilung \\
\hline$l_{i}$ & Ideelle Länge & $\tau_{n}$ & Nutteilung \\
\hline$N$ & Nutzahl & $\Phi_{\delta}$ & Luftspalttluss \\
\hline
\end{tabular}

Index 1 wird für Stator-Größen und der Index 2 für Rotor-Größen vereinbart. Der Index N steht für die Bemessungsgrößen. Im Rotor-Entwurf stehen der Index $r$ und der Index s jeweils für die Größen des Kurzschlussrings und des Stabs (Tab. 4).

Aus dem Grobentwurf können Statorwiderstand $R_{1}$ und Rotorwiderstand $R_{2}$ bzw. der auf die Statorseite bezogene Wert $R^{\prime}{ }_{2}$ bestimmt werden. $V_{\delta}, V_{\mathrm{z}}$ und $V_{\mathrm{rk}}$ sind magnetische Spannungsabfälle über dem Luftspalt, den Zähnen und dem Rücken. Je nach der Größe der Induktionen im Luftspalt $B_{\mathrm{m}}$, den Zähnen $B_{\mathrm{z}}$ und dem Rücken $B_{\mathrm{rk}}$ können die magnetischen Spannungsabfälle anhand der $B$ - $H$-Kurve des Elektroblechs ermittelt werden. Die Durchflutungsamplitude ergibt sich damit aus:

$$
\widehat{\Theta}_{\mathrm{p}}=V_{\delta}+V_{\mathrm{z} 1}+V_{\mathrm{rk} 1}+V_{\mathrm{z} 2}+V_{\mathrm{rk} 2}
$$

Für eine 3-phasige Maschine kann der Magnetisierungsstrom $I_{\mu}$ mit Gl. 9 berechnet werden. Dabei ist $p$ die Polpaarzahl. $w_{1}$ und $\xi_{1}$ sind die Windungszahl und der Wicklungsfaktor der Statorwicklung.

$$
I_{\mu}=\frac{\widehat{\Theta}_{\mathrm{p}} \cdot \pi \cdot p}{\sqrt{2} \cdot 3 \cdot w_{1} \cdot \xi_{1}}
$$

Die Hauptinduktivität $L_{\mathrm{h}}$ ergibt sich aus dem Magnetisierungsstrom, der Hauptfeldspannung $U_{\mathrm{h}}$ und der elektrischen Kreisfrequenz der Grundschwingung $\omega_{1}$.

$$
L_{\mathrm{h}}=\frac{U_{\mathrm{h}}}{\omega_{1} \cdot I_{\mu}}
$$

Neben der Hauptinduktivität können noch die Streuinduktivitäten des Stators $L_{\sigma 1}$ und des Rotors $L_{\sigma 2}$ mit Streuleitwerten ermittelt werden. Für die genauen Berechnungen sei auf [12] verwiesen.

Mit der oben vorgestellten Methode ist es möglich, die relevanten Parameter im Ersatzschaltbild (Abb. 5) nach der Eingabe von Bemessungsleistung, Bemessungsspannung, Bemessungsfrequenz und Bemessungsdrehzahl abzuschätzen. Selbstverständlich bestehen Abweichungen zwischen der Abschätzung und dem endgültigen Maschinenentwurf. In der Konzeptphase ist für den Vergleich aber vielmehr sinnvoller, in einer kurzen Zeit eine große Menge von brauchbaren Daten bereitzustellen.

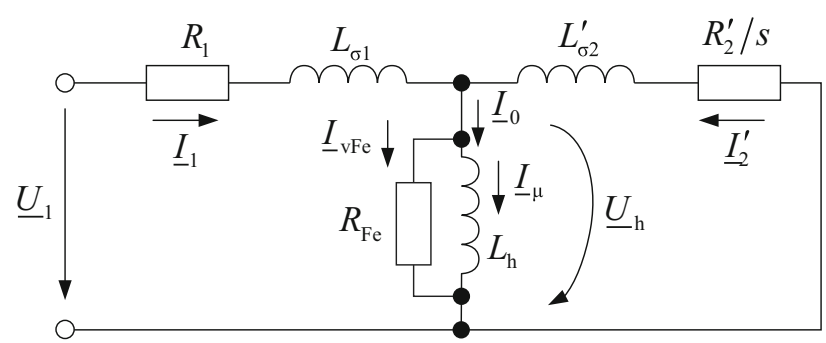

Abb. 5 Ersatzschaltbild der Asynchronmaschine 
Für die Verlustabschätzung kommt das Grundwellenmodell in Abb. 5 zum Einsatz, wobei die Einflüsse der Oberschwingungen zuerst vernachlässigt werden. Durch Drehstrom und Drehfeld werden Stromwärmeverluste $P_{\mathrm{vCu}}$ und Ummagnetisierungsverluste $P_{\mathrm{vFe}}$ hervorgerufen:

$$
\begin{aligned}
& P_{\mathrm{vCu}}=3 \cdot\left(R_{1} \cdot I_{1}^{2}+R_{2}^{\prime} \cdot I_{2}^{\prime 2}\right) \\
& P_{\mathrm{vFe}}=3 \cdot \frac{U_{\mathrm{h}}^{2}}{R_{\mathrm{Fe}}}
\end{aligned}
$$

$R_{\mathrm{Fe}}$ ist der Ersatzwiderstand für die Ummagnetisierungsverluste und lässt sich in zwei Anteile $R_{\mathrm{Fe} 0}$ für Wirbelstromverluste und $R_{\mathrm{Fel}}$ für Hystereseverluste aufteilen.

$$
R_{\mathrm{Fe}}=\left(\frac{1}{R_{\mathrm{Fe} 0}}+\frac{\omega_{1, \mathrm{~N}}}{\omega_{1} \cdot R_{\mathrm{Fe} 1}}\right)^{-1}
$$

Um $R_{\mathrm{Fe} 0}$ und $R_{\mathrm{Fe} 1} \mathrm{zu}$ bestimmen, wird die Formel der spezifischen Ummagnetisierungsverluste $p_{\mathrm{vFe}}$ nach Jordan [13] eingesetzt. Die Faktoren $k_{\mathrm{h}}$ und $k_{\mathrm{w}}$ sind abhängig von der Elektroblechsorte und können aus Herstellerangaben approximiert werden.

$$
p_{\mathrm{vFe}}=k_{\mathrm{h}} \cdot f \cdot \widehat{B}^{2}+k_{\mathrm{w}} \cdot f^{2} \cdot \widehat{B}^{2}
$$

Danach sind die Ummagnetisierungsverluste in den Zähnen $P_{\mathrm{vFe}, \mathrm{zl}}$ und dem Rücken $P_{\mathrm{vFe}, \mathrm{rkl}}$ berechenbar:

$$
\begin{aligned}
& P_{\mathrm{vFe}, \mathrm{zl}}=m_{\mathrm{Fe}, \mathrm{z} 1} \cdot\left(k_{\mathrm{h}} \cdot f_{1} \cdot \widehat{B}_{\mathrm{z} 1}^{2}+k_{\mathrm{w}} \cdot f_{1}^{2} \cdot \widehat{B}_{\mathrm{z} 1}^{2}\right) \\
& P_{\mathrm{vFe}, \mathrm{rk} 1}=m_{\mathrm{Fe}, \mathrm{rk} 1} \cdot\left(k_{\mathrm{h}} \cdot f_{1} \cdot \widehat{B}_{\mathrm{rk} 1}^{2}+k_{\mathrm{w}} \cdot f_{1}^{2} \cdot \widehat{B}_{\mathrm{rk} 1}^{2}\right)
\end{aligned}
$$

Die Masse der Zähne $m_{\mathrm{Fe}, \mathrm{zl}}$ und des Rückens $m_{\mathrm{Fe}, \mathrm{rk} 1}$ lassen sich mit der abgeschätzten Stator- und Rotorgeometrie ermitteln. Mit Gl. 12, (15) und (16) sind dann für verschiedene Frequenzen bzw. Hauptfeldspannungen die Ummagnetisierungsverluste $\mathrm{zu}$ berechnen, woraus $R_{\mathrm{Fe} 0}$ und $R_{\mathrm{Fe} 1}$ abgeleitet werden können.

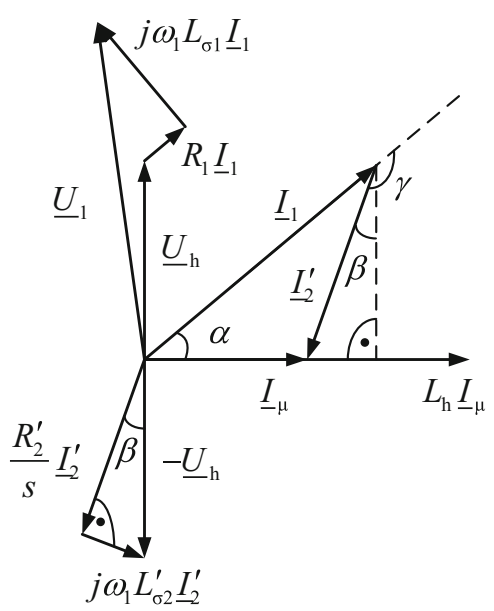

Um die Berechnung der Motorströme zu erklären, wird das Zeigerdiagramm in Abb. 6 verwendet. Das innere Motormoment ist abhängig vom Hauptfluss und Rotorstrom:

$$
M_{\mathrm{EM}}=3 \cdot p \cdot L_{\mathrm{h}} \cdot I_{\mu} \cdot I^{\prime}{ }_{2} \cdot \cos \beta
$$

Nach Zeigerdiagramm (Abb. 6) gibt es zwischen $I_{1}$, $I_{\mu}$ und $I_{2}^{\prime}$ folgenden Zusammenhang:

$$
I_{1}^{2}=\left(I_{2}^{\prime} \cdot \cos \beta\right)^{2}+\left(I_{\mu}+I_{2}^{\prime} \cdot \sin \beta\right)^{2}
$$

mit

$$
\sin \beta=\frac{\omega_{1} \cdot L_{\sigma 2}^{\prime} \cdot I_{2}^{\prime}}{U_{\mathrm{h}}}=\frac{\omega_{1} \cdot L_{\sigma 2}^{\prime} \cdot I_{2}^{\prime}{ }_{2}}{\omega_{1} \cdot L_{\mathrm{h}} \cdot I_{\mu}}=\frac{L_{\sigma 2}^{\prime} \cdot I_{2}^{\prime}}{L_{\mathrm{h}} \cdot I_{\mu}}
$$

Durch Einsetzen von (19) in (18) folgt:

$$
I_{1}^{2}=I_{\mu}^{2}+\left(2 \cdot \frac{L_{\sigma 2}^{\prime}}{L_{\mathrm{h}}}+1\right) \cdot I_{2}^{\prime 2}
$$

Für Motorsteuerverfahren wird oft die MTPA-Methode (Maximum-Torque-per-Ampere) verwendet, damit der Statorstrom $I_{1}$ bzw. die statorseitigen Stromwärmeverluste minimiert werden können. $I_{\mu}$ und $I_{2}{ }_{2}$ lassen sich dann mit Hilfe der Lagrange-Funktion ermitteln:

$$
\begin{aligned}
& \mathrm{L}=3 p L_{\mathrm{h}} I_{\mu} I_{2}^{\prime} \cos \beta+\lambda\left(I_{1}^{2}-I_{\mu}^{2}-\left(2 \cdot \frac{L_{\sigma 2}^{\prime}}{L_{\mathrm{h}}}+1\right) I_{2}^{\prime 2}\right) \\
& \frac{\partial \mathrm{L}}{\partial I^{\prime}{ }_{2}}=\frac{\partial \mathrm{L}}{\partial I_{\mu}}=\frac{\partial \mathrm{L}}{\partial \lambda}=0
\end{aligned}
$$

Um das Lösen der Lagrange-Funktion zu erleichtern, wird folgende Annahme getroffen:

$$
L_{\sigma 2}^{\prime} \ll L_{\mathrm{h}}
$$

$I_{1}, I_{\mu}$ und $I_{2}^{\prime}$ ergeben sich somit aus:

$$
\begin{aligned}
& I_{\mu}=I_{2}^{\prime}=\frac{\sqrt{2}}{2} I_{1} \\
& I_{1}=\sqrt{\frac{2 \cdot M_{\mathrm{EM}}}{3 \cdot p \cdot L_{\mathrm{h}}}}
\end{aligned}
$$

Wenn das Maximum der Motorspannung erreicht wird, wird zu einer maximalen Spannungsausnutzung das Steuerverfahren von MTPA zu MTPV (MaximumTorque-per-Voltage) übergegangen. Die Motorströme bei MTPV werden analog zu MTPA berechnet:

$$
\begin{aligned}
I_{\mu} & \approx \frac{U_{1, \max }}{\omega_{1} \cdot L_{\mathrm{h}}}, I^{\prime}{ }_{2} \approx \frac{\omega_{1} \cdot M_{\mathrm{EM}}}{3 \cdot p \cdot U_{1, \max }} \\
I_{1} & \approx \sqrt{\left(\frac{U_{1, \max }}{\omega_{1} \cdot L_{\mathrm{h}}}\right)^{2}+\left(\frac{\omega_{1} \cdot M_{\mathrm{EM}}}{3 \cdot p \cdot U_{1, \max }}\right)^{2}}
\end{aligned}
$$

Abb. 6 Zeigerdiagramm der Asynchronmaschine 


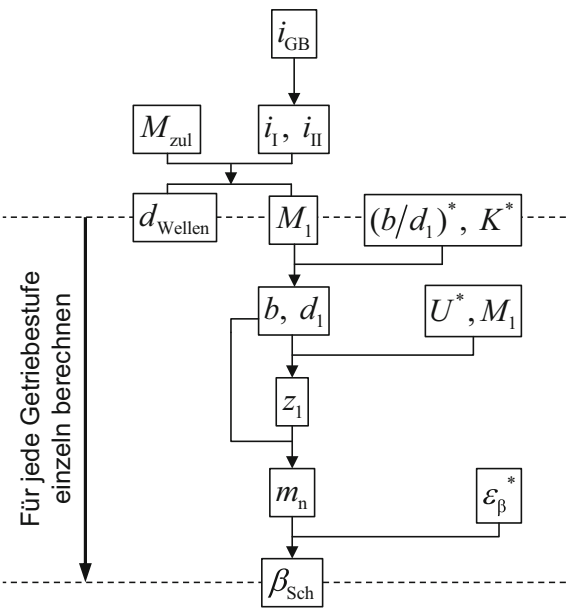

Abb. 7 Grobentwurf eines 1-Gang-Getriebes

\section{Abschätzung der Getriebe-Geometrie}

Im Vergleich zu Verbrennungsmotoren können Elektromotoren üblicherweise in einem größeren Drehzahlspektrum mit relativ hohem Wirkungsgrad arbeiten. Das hat zur Folge, dass statt eines klassischen mehrgängigen Fahrzeuggetriebes lediglich eine Reduktionsstufe bzw. 1-Gang-Getriebe in Elektrofahrzeugen eingebaut sind. Die Verluste eines 1-GangGetriebes lassen sich vor allem in Verzahnungsverlusten, Lagerverlusten und Dichtungsverlusten unterscheiden. Um diese Verluste mit empirischen Formeln [9] einzeln zu berechnen, müssen einige geometrische Parameter von den Wellen, den Zahnrädern und den Lagern bekannt sein. Angesichts der relativ einfachen Struktur eines 1-Gang-Getriebes ist es möglich, diese geometrischen Parameter anhand von einem generellen Getriebeentwurf nach Niemann [14] abzuschätzen. Der Entwurfsprozess wird in Abb. 7 dargestellt. Für die Parameter, die mit * gekennzeichnet sind, werden zuerst Richtwerte anhand von [14] ausgewählt. Der Index 1 steht für die Größen des Ritzels von einem Zahnradpaar.

Laut Niemann ist eine zweistufige Ausführung des Getriebes wirtschaftlich von Vorteil, wenn die Getriebeübersetzung $i_{\mathrm{GB}}$ in einem Bereich zwischen 6 und 35 liegt, welcher üblich für Fahrzeuganwendungen ist. Die Übersetzung einzelner Stufen lässt sich anhand von [14] mit (28) berechnen:

$$
i_{\mathrm{I}}=0,8 \cdot\left(i_{\mathrm{GB}}\right)^{\frac{2}{3}}, i_{\mathrm{II}}=\frac{i_{\mathrm{GB}}}{i_{\mathrm{I}}}
$$

Aus den Übersetzungen und dem zulässigen Getriebe-Eingangsmoment $M_{\text {zul }}$ ergeben sich die zulässigen Wellenmomente $M_{1}$. Die Durchmesser einzelner Wellen $d_{\text {wellen }}$ werden gemäß (29) berechnet, wo- bei $\tau_{t, \text { zul }}$ die zulässige Torsionsspannung der Werkstoffe ist.

$$
d_{\text {Wellen }}=\sqrt[3]{\frac{16}{\pi} \cdot \frac{M_{1}}{\tau_{\mathrm{t}, \mathrm{zul}}}}
$$

Um die Bestimmung von Zahnradbreite $b$, Teilkreisdurchmesser $d_{1}$ und Zähnezahl $z_{1}$ zu vereinfachen, werden Faktor $K$ und $U$ von Niemann eingeführt, deren Referenzwerte in [14] abhängig von Getriebeanwendungen angegeben sind.

$$
\begin{aligned}
& d_{1}=\sqrt[3]{\frac{2000 M_{1}}{K \cdot\left(b / d_{1}\right)} \cdot \frac{i+1}{i}} \\
& z_{1}=U \cdot \frac{b \cdot d_{1}^{2}}{2000 \cdot M_{1}}
\end{aligned}
$$

Das Normalmodul $m_{\mathrm{n}}$ ergibt sich somit aus:

$$
m_{\mathrm{n}}=\frac{d_{1}}{z_{1}}
$$

Für eine gute Laufruhe sind die Zähne der Zahnräder meistens um einen Winkel zur Rotationsachse geschrägt. Der Schrägungswinkel $\beta_{\text {Sch }}$ lässt sich nach

$$
\sin \beta_{\text {Sch }}=\frac{m_{\mathrm{n}} \cdot \pi \cdot \varepsilon_{\beta}}{b}
$$

bestimmen, wobei $\varepsilon_{\beta}$ die Sprungüberdeckung ist. Hinsichtlich Laufruhe und Axialkraft ist nach [15] eine Sprungüberdeckung zwischen 1,0 und 1,2 bevorzugt.

\section{Approximation der Verlustkennfelder}

Für die Zielstellung dieser Arbeit ist eine ganzheitliche Betrachtung des Antriebsstrangs erforderlich. Die gesamte Verlustleistung des Antriebsstrangs lässt sich über den einzelnen Verlustleistungen der Komponenten last- und drehzahlabhängig bestimmen. Zwischen der Verlustleistung und dem Drehmoment bzw. der Drehzahl bestehen nichtlineare und stückweise $\mathrm{Zu}$ sammenhänge, die vor allem auf physikalische Eigen-

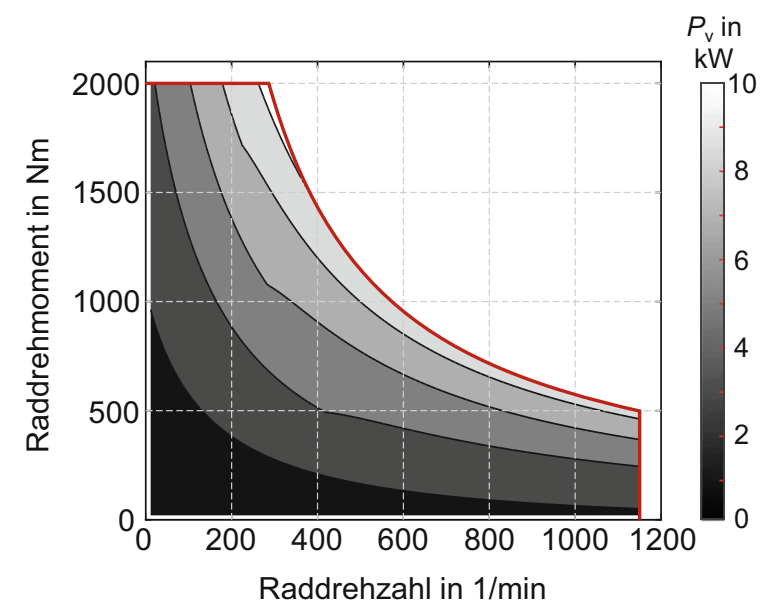

Abb. 8 Verlustkennfeld eines Beispiel-Antriebsstrangs 
schaften und Steuerungsstrategien der Komponenten zurückzuführen sind. Für die Untersuchung dieser Zusammenhänge sind Verlustkennfelder ein wichtiges Mittel. In Abb. 8 ist das Verlustkennfeld eines Beispiel-Antriebsstrangs dargestellt.

Um die nachfolgende Herleitung des Auswahldiagramms zu vereinfachen, wird das Verlustkennfeld zuerst mit einem nichtlinearen Polynom 2. Grades nach (34) approximiert.

$$
P_{\mathrm{V}}(n, M)=k_{1} n^{2}+k_{2} n+k_{3} n M+k_{4} M+k_{5} M^{2}
$$

Dabei ist $n$ die Raddrehzahl, $M$ das Raddrehmoment. $k_{1}$ bis $k_{5}$ sind die Faktoren des Polynoms und abhängig von Parametern eines Antriebsstrangs.

In dieser Arbeit wird für jeden Fahrzyklus noch ein äquivalenter Betriebspunkt ( $n_{\mathrm{eq}}, M_{\mathrm{eq}}$ ) eingeführt. Für den Betriebspunkt gilt Gl. 35,

$$
P_{\mathrm{v}}\left(n_{\mathrm{eq}}, M_{\mathrm{eq}}\right) \cdot \sum_{\mathrm{i}=1}^{N} t_{\mathrm{i}}=\sum_{\mathrm{i}=1}^{N} P_{\mathrm{v}}\left(n_{\mathrm{i}}, M_{\mathrm{i}}\right) \cdot t_{\mathrm{i}}
$$

wobei $N$ die Anzahl der Betriebspunkte eines Fahrzyklus und $t$ die Zeitdauer einzelner Betriebspunkte sind. Es handelt sich um den mittleren Betriebszustand eines Fahrzyklus bzgl. der Zyklus-Verlustwärme des Antriebsstrangs. Diese obengenannte Approximation dient hier nur zur Bestimmung des Äquivalenzpunkts und kann die Berechnungskomplexität stark reduzieren. Da das Polynom (34) ohnehin nicht zur Verlustberechnung eingesetzt wird, spielen die Abweichungen zwischen dem realen Verlustkennfeld und dem approximierten Kennfeld hier kaum eine Rolle.

Es ist zu erkennen, dass die Werte $n_{\mathrm{eq}}$ und $M_{\mathrm{eq}}$ nicht nur vom Fahrzyklus, sondern auch von den Faktoren $k_{1}$ bis $k_{5}$ beeinflusst werden. Aus Gl. 36-38 kann jedoch ein von den Polynom-Faktoren unabhängiges Intervall bestimmt werden, in dem sich der äquivalente Betriebspunkt befindet.

$$
\begin{aligned}
& n_{\mathrm{I}}=\sqrt{\frac{\sum_{\mathrm{i}=1}^{N} k_{1} \cdot n_{\mathrm{i}}^{2} \cdot t_{\mathrm{i}}}{\sum_{\mathrm{i}=1}^{N} k_{1} \cdot t_{\mathrm{i}}}}, M_{\mathrm{I}}=\sqrt{\frac{\sum_{\mathrm{i}=1}^{N} k_{5} \cdot M_{\mathrm{i}}^{2} \cdot t_{\mathrm{i}}}{\sum_{\mathrm{i}=1}^{N} k_{5} \cdot t_{\mathrm{i}}}} \\
& n_{\mathrm{II}}=\frac{\sum_{\mathrm{i}=1}^{N} k_{2} \cdot n_{\mathrm{i}} \cdot t_{\mathrm{i}}}{\sum_{\mathrm{i}=1}^{N} k_{2} \cdot t_{\mathrm{i}}}, M_{\mathrm{II}}=\frac{\sum_{\mathrm{i}=1}^{N} k_{4} \cdot M_{\mathrm{i}} \cdot t_{\mathrm{i}}}{\sum_{\mathrm{i}=1}^{N} k_{4} \cdot t_{\mathrm{i}}} \\
& n_{\mathrm{III}}=\frac{\sum_{\mathrm{i}=1}^{N} k_{3} \cdot M_{\mathrm{i}} \cdot n_{\mathrm{i}} \cdot t_{\mathrm{i}}}{\sum_{\mathrm{i}=1}^{N} k_{3} \cdot M_{\mathrm{i}} \cdot t_{\mathrm{i}}}, M_{\mathrm{III}}=\frac{\sum_{\mathrm{i}=1}^{N} k_{3} \cdot M_{\mathrm{i}} \cdot n_{\mathrm{i}} \cdot t_{\mathrm{i}}}{\sum_{\mathrm{i}=1}^{N} k_{3} \cdot n_{\mathrm{i}} \cdot t_{\mathrm{i}}}
\end{aligned}
$$

Tab. 5 Vorgegebene Fahrzeugdaten

\begin{tabular}{|l|l|}
\hline Parameter & Werte \\
\hline Luftwiderstandsbeiwert & 0,32 \\
\hline Stirnfläche & $2,1 \mathrm{~m}^{2}$ \\
\hline Rollwiderstandsbeiwert & 0,01 \\
\hline Drehmassenzuschlagfaktor & 1,05 \\
\hline Dynamischer Reifenradius & $0,3 \mathrm{~m}$ \\
\hline Leergewicht & $1235 \mathrm{~kg}$ \\
\hline Beschleunigungszeit 0-100 km/h & $<11,9 \mathrm{~s}$ \\
\hline Höchstgeschwindigkeit & $>130 \mathrm{~km} / \mathrm{h}$ \\
\hline Steigfähigkeit beim Anfahren & $>35 \%$ \\
\hline Reichweite (WLTP 3) & $>200 \mathrm{~km}$ \\
\hline
\end{tabular}

Das Intervall nach Gl. 39 und (40) wird als Äquivalenzbereich des Fahrzyklus bezeichnet.

$$
\begin{aligned}
& n_{\mathrm{eq}} \in\left[\min \left(n_{\mathrm{I}}, n_{\mathrm{II}}, n_{\mathrm{III}}\right), \max \left(n_{\mathrm{I}}, n_{\mathrm{II}}, n_{\mathrm{III}}\right)\right] \\
& M_{\mathrm{eq}} \in\left[\min \left(M_{\mathrm{I}}, M_{\mathrm{II}}, M_{\mathrm{III}}\right), \max \left(M_{\mathrm{I}}, M_{\mathrm{II}}, M_{\mathrm{III}}\right)\right]
\end{aligned}
$$

Die Herleitung des Äquivalenzbereichs ist sinnvoll, da dadurch der äquivalente Betriebszustand eines Fahrzyklus von konkreten Antriebsstrang-Konfigurationen entkoppelt und nur abhängig von Fahrprofilen des Fahrzyklus bestimmt werden kann.

\section{Auswahldiagramm für energetische Bewertung}

Basierend auf einem Beispielfahrzeug wird das Auswahldiagramm vorgestellt. Die Grundparameter des Fahrzeugs werden in Tab. 5 vorgegeben. In diesem

\begin{tabular}{|c|c|c|c|}
\hline & EM1 & EM2 & EM3 \\
\hline$P_{\text {mech,N }}$ & $60 \mathrm{~kW}$ & $60 \mathrm{~kW}$ & $60 \mathrm{~kW}$ \\
\hline$M_{N}$ & $386 \mathrm{Nm}$ & $193 \mathrm{Nm}$ & $96 \mathrm{Nm}$ \\
\hline$U_{L L, N}$ & $400 \mathrm{~V}, \mathrm{Y}$ & $400 \mathrm{~V}, \mathrm{Y}$ & $400 \mathrm{~V}, \mathrm{Y}$ \\
\hline IN & $102 \mathrm{~A}$ & $109 \mathrm{~A}$ & $112 \mathrm{~A}$ \\
\hline$R_{1}\left(20^{\circ} \mathrm{C}\right)$ & $59 \mathrm{~m} \Omega$ & $43 \mathrm{~m} \Omega$ & $29 \mathrm{~m} \Omega$ \\
\hline$R^{\prime}{ }_{2}\left(20^{\circ} \mathrm{C}\right)$ & $49 \mathrm{~m} \Omega$ & $35 \mathrm{~m} \Omega$ & $23 \mathrm{~m} \Omega$ \\
\hline$L_{\mathrm{h}, \mathrm{N}}$ & $21,4 \mathrm{mH}$ & $11,1 \mathrm{mH}$ & $4,8 \mathrm{mH}$ \\
\hline$R_{\mathrm{Fe} 0}$ & $1784 \Omega$ & $945 \Omega$ & $457 \Omega$ \\
\hline$R_{\mathrm{Fe} 1}$ & $343 \Omega$ & $364 \Omega$ & $352 \Omega$ \\
\hline$p$ & 2 & 2 & 2 \\
\hline$l_{i}$ & $291 \mathrm{~mm}$ & $231 \mathrm{~mm}$ & $183 \mathrm{~mm}$ \\
\hline$D$ & $185 \mathrm{~mm}$ & $147 \mathrm{~mm}$ & $117 \mathrm{~mm}$ \\
\hline w1 & 40 & 36 & 30 \\
\hline$N_{1}$ & 48 & 36 & 36 \\
\hline $\mathrm{N}_{2}$ & 40 & 28 & 28 \\
\hline$i_{\mathrm{GB}}$ & 3,24 & 6,42 & 12,95 \\
\hline
\end{tabular}
Beispiel sind verschiedene Kombinationen von Motor und Getriebe zu untersuchen. Hinsichtlich der erforderlichen Fahrzeugeigenschaften in Tab. 5 kann der Antriebsstrang mit einem maximalen Radmoment von $1200 \mathrm{Nm}$, einer maximalen Raddrehzahl von 1150 $1 /$ min und einer maximalen Antriebsleistung von 
Tab. 7 Batterie und Wechselrichter

\begin{tabular}{|l|l|}
\hline Batterie & $\begin{array}{l}31 \mathrm{kWh}, 336 \mathrm{~V}, \text { Kokam 31 Ah-Zelle } \\
\text { SLPB78216216H }\end{array}$ \\
\hline 2-Level-Wechselrichter & $\begin{array}{l}\text { IGBT, 650V, 600A, } \\
\text { Infineon FF600R07ME4 }\end{array}$
\end{tabular}

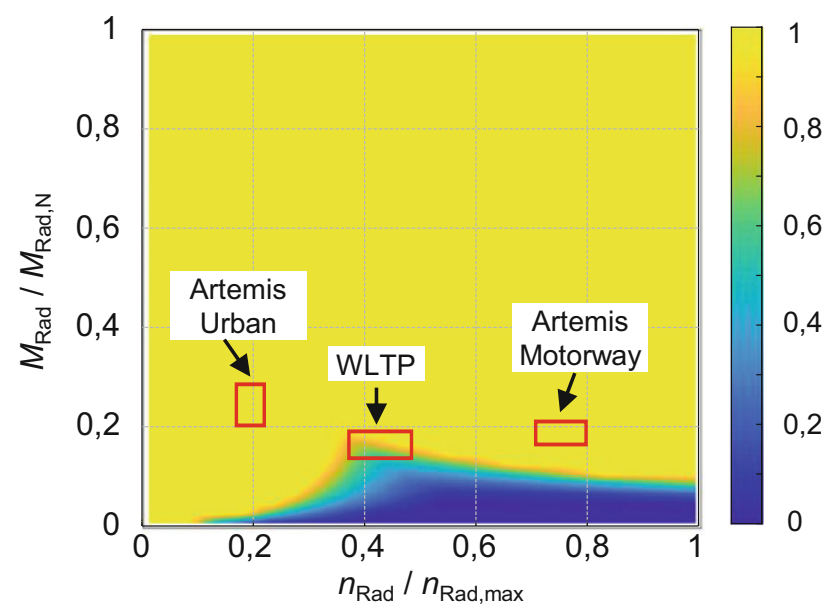

Abb. 9 Auswahldiagramm der Konfiguration 1, ausgerüstet mit EM1 (bei Motortemperatur $120^{\circ} \mathrm{C}$ )

$60 \mathrm{~kW}$ ausgelegt werden. Als Antriebsmotor werden beispielsweise drei Asynchronmaschinen mit unterschiedlichen Bemessungsmomenten konzipiert. Die Motordaten sind in Tab. 6 dargestellt. Für EM1, EM2 und EM3 wird hinsichtlich des maximalen Radmoments jeweils eine Übersetzung von 3,24, 6,42 und 12,95 gewählt. $\mathrm{Zu}$ beachten ist, dass eine energetische Optimierung dieser Getriebeübersetzungen hier nicht durchgeführt wird. Verschiedene Fahrzyklen führen in der Regel zu unterschiedlichen Optimierungsergebnissen, was die energetische Optimierung der Übersetzung nicht mehr zielführend macht. Weitere Daten des Antriebsstrangs sind aus Tab. $7 \mathrm{zu}$ entnehmen.

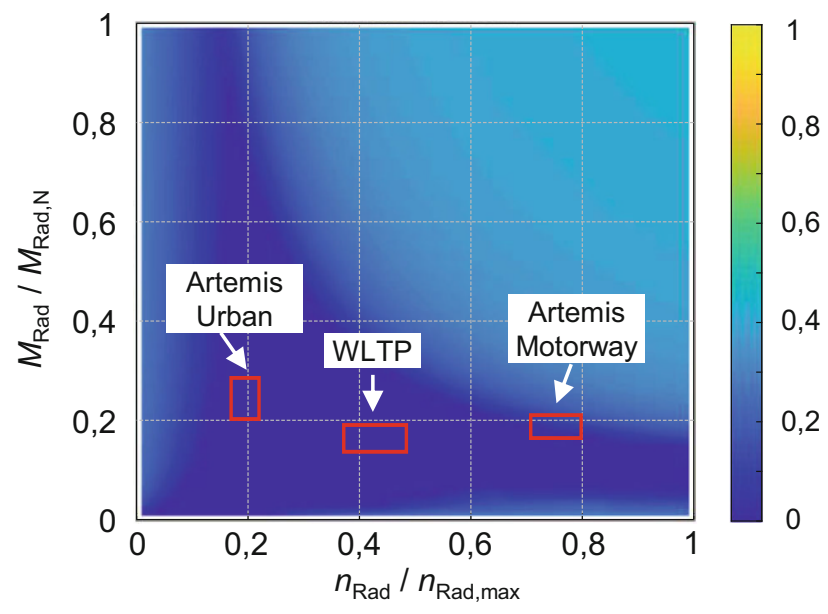

Abb. 10 Auswahldiagramm der Konfiguration 2, ausgerüstet mit EM2 (bei Motortemperatur $120^{\circ} \mathrm{C}$ )

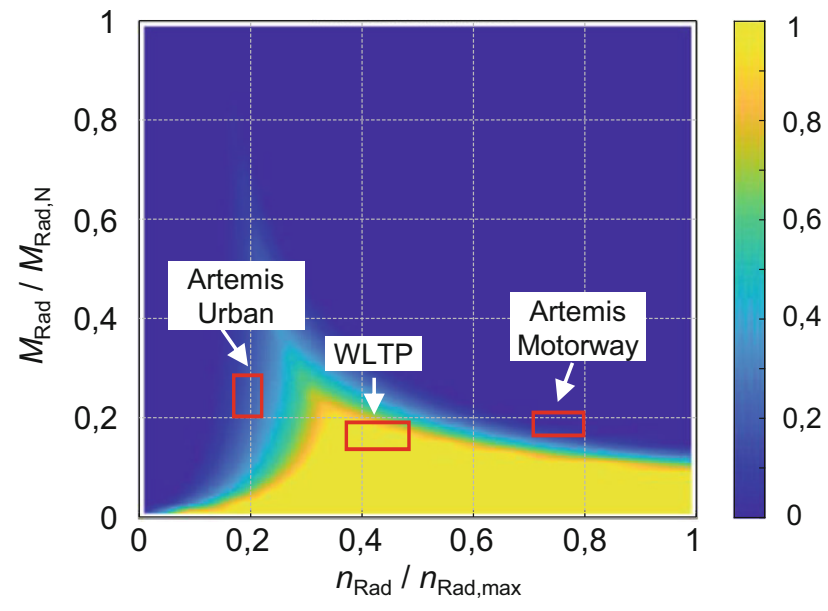

Abb. 11 Auswahldiagramm der Konfiguration 3, ausgerüstet mit EM3 (bei Motortemperatur $120^{\circ} \mathrm{C}$ )

In Abb. 9 und 10 und 11 werden die Auswahldiagramme der drei Antriebskonfigurationen dargestellt. Die Farben in einem Auswahldiagramm entsprechen einem normierten Intervall von 0 bis 1 und weisen darauf hin, ob die Konfiguration bei einem bestimmten Betriebszustand energieeffizienter als andere Konfigurationen ist. Dabei ist 0 das energieeffizienteste, 1 das energieineffizienteste. Je dunkler die Farbe ist, desto effizienter ist die Konfiguration gegenüber den anderen.

Da EM3 kleinere Stator- und Rotorwiderstände hat, sind seine Stromwärmeverluste viel geringer als bei EM1 und EM2. Das hat zur Folge, dass Konfiguration 3 bei hohen Drehmomenten, wo die Stromwärmeverluste signifikant sind, effizienter als die anderen Konfigurationen ist. Demgegenüber ist Konfiguration 1 lediglich bei hoher Drehzahl und sehr geringen Drehmomenten effizienter als die anderen, da nur in diesen Bereichen der Vorteil der geringen Ummagnetisierungsverluste von EM1 zum Tragen kommt. Konfiguration 2 ist in den meisten Bereichen zwar nicht das energieeffizienteste aller Konfigurationen, aber immerhin auch nicht das energieineffizienteste. Deswegen kann Konfiguration 2 als eine ausgeglichene Lösung betrachtet werden.

Um die Nutzung der Auswahldiagramme zu erklären, werden jeweils ein Stadtzyklus, ein Autobahnzyklus und ein gemischter Zyklus ausgewählt. Ihre Äquivalenzbereiche werden in den Auswahldiagrammen mit roten Kästen gekennzeichnet. Da diese Fahrzyklen verschiedene Fahrszenarien repräsentieren, unterscheiden sich ihre Äquivalenzbereiche voneinander deutlich. Mit Hilfe der Auswahldiagramme lassen sich Prognosen anstellen, bei der Konfiguration 3 für Artemis-Motorway und Konfiguration 2 für Artemis-Urban und WLTP energieoptimal sind.

Zur Nachprüfung der Prognose wird für jede Konfiguration die Zyklus-Verlustwärme von Batterie, Wechselrichter, Motor und Getriebe simulativ ermittelt und in Abb. 12 dargestellt. Die grundsätzliche Modellie- 


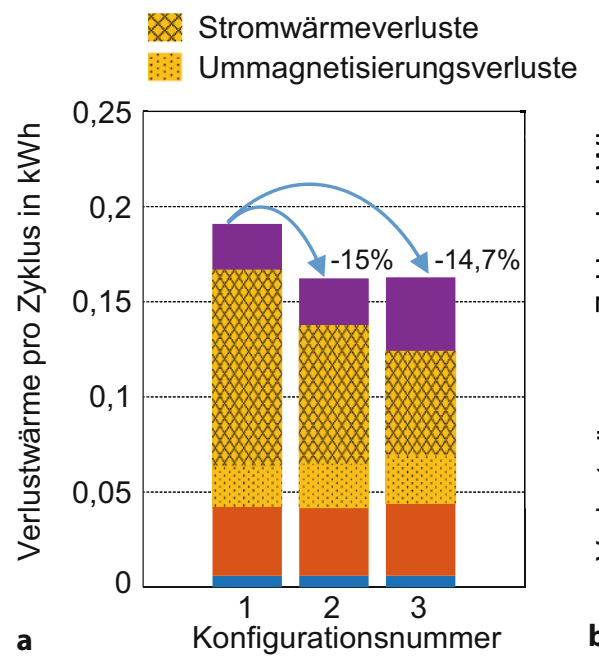

Effizienzgrad (je nach Zyklus und Konfiguration)

GB: $95 \%$ - $97 \%$, EM: $89 \%$ - $95 \%$, WR: $96 \%$ - $98 \%$, BAT: $96 \%-99 \%$
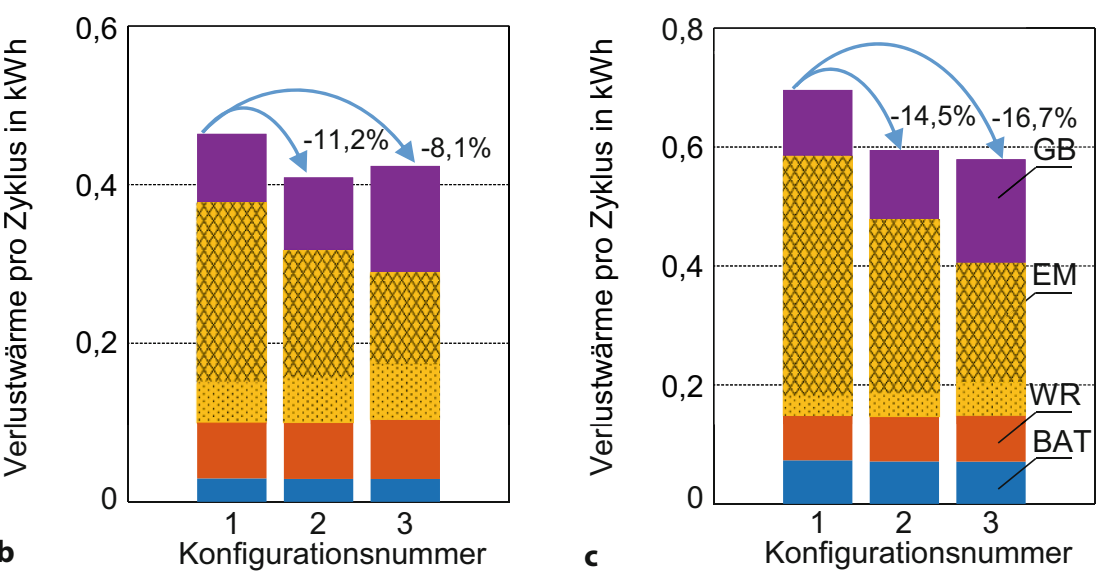

Abb. 12 Simulierte Verlustwärme bei Motortemperatur $120^{\circ} \mathrm{C}$, a für Artemis-Urban, b für WLTP, c für Artemis-Motorway. (GB Getriebe, EM Elektromotor, WR Wechselrichter, BAT Batterie)

rung wurde in [16] bei einem Antrieb für Stadtbusse überprüft. Im Bereich kleiner Motorströme ist die berechnete Motor- und Wechselrichter-Verlustleistung kleiner als die gemessene. Das ist auf das Grundwellenmodell des Motors zurückzuführen. Bei kleiner Grundschwingung haben die Oberschwingungsverluste einen verhältnismäßig hohen Anteil an den Gesamtverlusten. Hinsichtlich des Energieverbrauchs wird bei Konfiguration 1 ein Zyklusverbrauch von 2,6 kWh für WLTP-Zyklus berechnet, der mit dem in [17] angegeben WLTP-Zyklusverbrauch von $3 \mathrm{kWh}$ vergleichbar ist. Die Berechnungen in [17] basieren auf einem Kleinwagen mit einem 1-Gang-Getriebe und einer ASM von $100 \mathrm{~kW}$, welche höher als die Motorleistung in dieser Arbeit ist.

In Abb. 12 werden die Konfigurationen hinsichtlich der Energieeffizienz miteinander verglichen, woraus die energieeffizienteste Konfiguration für jeden Fahrzyklus bestimmt wird. Die Ergebnisse stimmen mit der Vorhersage der Auswahldiagramme überein.

\section{Zusammenfassung}

In dieser Arbeit wurde eine Methode vorgestellt, um in einem frühen Entwicklungsstadium energieeffiziente Antriebskonfigurationen für Elektrofahrzeuge auszuwählen. Hierzu werden Antriebskomponenten wie Batterie, Wechselrichter, Elektromotor und Getriebe einbezogen. Für einen energetischen Vergleich der Konfigurationen kommen physikalische Modelle der Komponenten zum Einsatz. Da beim Konzeptvergleich die endgültigen Komponentenauslegungen häufig noch nicht festgelegt sind, ist es sinnvoll, Komponentenparameter basierend auf Herstellerangaben und generellem Entwurf abzuschätzen. Die in dieser Arbeit beschriebene Vorgehensweise ermöglicht eine schnelle Bereitstellung von brauchbaren Komponentendaten.
Um eine Vorhersage der energieoptimalen Antriebskonfiguration für beliebige Einsatzprofile zu ermöglichen, wird ein Auswahldiagramm konzipiert, in dem einzelne Fahrzyklen durch ihren Äquivalenzbereich repräsentiert werden. Die überschaubare Darstellung im Auswahldiagramm weist darauf hin, in welchem Umfang einzelne Konfigurationen die effizienteste Lösung sind.

Danksagung Das Projekt FVA 886 I „Auswahlsystematik für energieeffiziente Antriebsstränge in rein elektrischen Straßenfahrzeugen“ wurde von der „Forschungsvereinigung Antriebstechnik e.V.“ (FVA) aus Eigenmitteln gefördert.

Funding Open Access funding enabled and organized by Projekt DEAL.

Open Access Dieser Artikel wird unter der Creative Commons Namensnennung 4.0 International Lizenz veröffentlicht, welche die Nutzung, Vervielfältigung, Bearbeitung, Verbreitung und Wiedergabe in jeglichem Medium und Format erlaubt, sofern Sie den/die ursprünglichen Autor(en) und die Quelle ordnungsgemäß nennen, einen Link zur Creative Commons Lizenz beifügen und angeben, ob Änderungen vorgenommen wurden.

Die in diesem Artikel enthaltenen Bilder und sonstiges Drittmaterial unterliegen ebenfalls der genannten Creative Commons Lizenz, sofern sich aus der Abbildungslegende nichts anderes ergibt. Sofern das betreffende Material nicht unter der genannten Creative Commons Lizenz steht und die betreffende Handlung nicht nach gesetzlichen Vorschriften erlaubt ist, ist für die oben aufgeführten Weiterverwendungen des Materials die Einwilligung des jeweiligen Rechteinhabers einzuholen.

Weitere Details zur Lizenz entnehmen Sie bitte der Lizenzinformation auf http://creativecommons.org/licenses/by/4. $0 /$ deed.de.

\section{Literatur}

1. Kreim A (2015) Modellierung und Parameteroptimierung einer permanenterregten Synchronmaschine unter Be- 
rücksichtigung von Lastzyklen. Dissertation. Technische UniversitätBerlin

2. Windisch T, Hofmann W (2018) A Novel Approach to MTPA Tracking Control of AC Drives in Vehicle Propulsion Systems. IEEE Transactions on Vehicular Technology 67(10):9294-9302

3. Eghtessad M (2015) Optimale Antriebsstrangkonfigurationen für Elektrofahrzeuge. Dissertation. Technische UniversitätBraunschweig

4. ReupoldP (2014) LösungsraumanalysefürHauptantriebsstränge in batterieelektrischen Straßenfahrzeugen. Dissertation. Technische Universität München

5. Frenzel I, Jarass J, Tromer S, Lenz B (2015) Erstnutzer von Elektrofahrzeugen in Deutschland. DLR Forschungsbericht

6. Jian L (2018) Listening to the voices of customers. China Machine Press

7. BMVI (2019) Mobilität in Deutschland. BMVI Forschungsbericht

8. Ou S, Yu R, Lin Z, Ren H, He X, Przesmitzki S, Bouchard J (2019) Intensity and daily pattern of passenger vehicle use byregion and classinChina: estimation andimplicationsfor energy use and electrification. Mitigation and Adaptation Strategies for Global Change 25(3):307-327

9. Yuan S, Hofmann W (2020) Data Sheets based Design of an Energy Efficient Powertrain of Electric Vehicles. In: 15th International Conference on Ecological Vehicles and Renewable Energies. S 1-9

10. Wintrich A, Nicolai U, Tursky W, Reimann T (2010) Applikationshandbuch Leistungshalbleiter. ISLE Verlag, Ilmenau

11. Dieckerhoff S, Bernet S, Krug D (2005) Power loss-oriented evaluation of high voltage IGBTs and multilevel converters in transformerless traction applications. IEEETransactions on Power Electronics 20(6):1328-1336

12. Müller G, Vogt K, Ponick B (2007) Berechnung elektrischer Maschinen. Wiley-VCH, Berlin

13. Jordan H (1924) Die ferromagnetischen Konstanten für schwache Wechselfelder. Elektrische Nachrichten Technik $1: 7-29$

14. Niemann G, Winter H (2003) Maschinenelemente Band II: Getriebe allgemein, Zahnradgetriebe - Grundlagen, Stirnradgetriebe. Springer, Berlin, Heidelberg.

15. Wittel H, Muhs D, Jannasch D, Voßiek J (2015) Roloff/Matek Maschinenelemente-Normung, Berechnung, Gestaltung. SpringerVieweg, Wiesbaden
16. Windisch T (2018) Energieeffiziente Antriebsregelung für hochausgenutzte Drehstrommotoren in elektrisch angetriebenen Fahrzeugen. Dissertation. Technische UniversitätDresden

17. Reichert U (2019) Eine Methode zur Auswahl von Standgetrieben für Antriebsstränge von Elektrofahrzeugen mit Zentralantrieb. Dissertation. Karlsruher Institut für Technologie

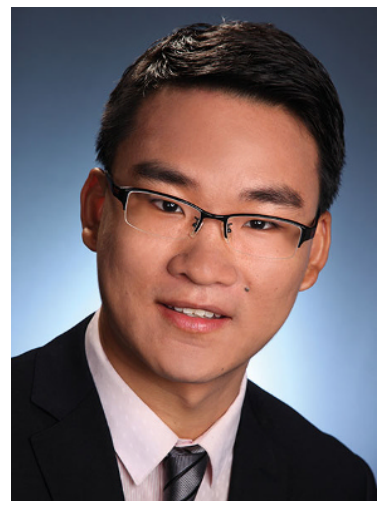

Shaohui Yuan, hat 2016 sein Masterstudium im Bereich Kraftfahrzeugtechnik an der TU Braunschweig absolviert. Seit 2017 ist er als Promovend und wissenschaftlicher Mitarbeiter am Lehrstuhl für elektrische Maschinen und Antriebe der TU Dresden tätig. $\mathrm{Zu}$ seinen Forschungsinteressen gehören die analytische Verlustberechnung der elektrischen Antriebsstränge und die Optimierung der Antriebsstrangauslegung für Elektrofahrzeuge.

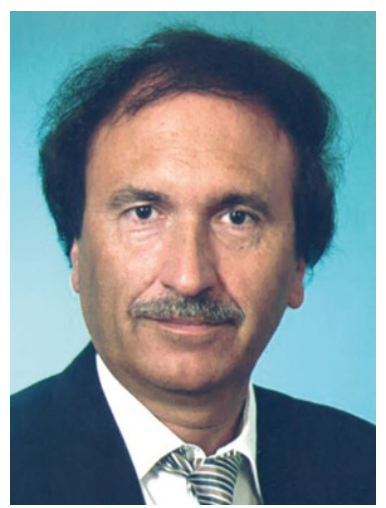

Wilfried Hofmann, studierte Elektrotechnik an der TU Dresden, Diplomabschluss 1978, Promotion 1984. Industrietätigkeit als Entwicklungsingenieur bei Elektroprojekt- und Anlagenbau Berlin 1982-1988/89. C4-Professorfür Elektrische Maschinen und Antriebe an der TU Chemnitz 1992-2007, W3-Professor für Elektrische Maschinen und Antriebe an der TU Dresden 2007-2020, danach Seniorprofessor. Forschungsschwerpunkte sind $u$.a. energieeffiziente Industrie- und Fahrantriebe von der Elektromaschine über die Umrichtertechnikbis zur Regelung. 